\title{
BEAM-BEAM COMPENSATION IN TEVATRON: STATUS REPORT
}

\author{
V. Shiltsev, G.Kuznetsov, N. Solyak, D. Wildman, X.L. Zhang, FNAL, Batavia, IL 60510, USA \\ Yu.Alexahin, JINR, Dubna, Russia; and K.Bishofberger, UCLA, Los-Angeles, CA 90095, USA
}

\begin{abstract}
The project of beam-beam compensation (BBC) in the Tevatron using electron beams [1] has passed a successful first step in experimental studies. The first Tevatron electron lens (TEL) has been installed in the Tevatron, commissioned, and demonstrated the theoretically predicted shift of betatron frequencies of a high energy proton beam due to a high current low energy electron beam. After the first series of studies in March-April 2001 (total of 7 shifts), we achieved tuneshifts of $980 \mathrm{GeV}$ protons of about $d Q=+0.007$ with some 3 A of the electron beam current while the proton lifetime was in the range of 10 hours (some 24 hours at the best). Future work will include diagnostics improvement, beam studies with antiprotons, and fabrication of the 2nd TEL.
\end{abstract}

\section{BRIEF DECRIPTION OF BBC AND TEL}

In the Tevatron, the antiproton bunches suffer a tuneshift due to their interactions with the more intense proton bunches. In multibunch operation, the tuneshifts vary from antiproton bunch to antiproton bunch, leading to an effective spread in tune. An electron lens, consisting of a short, low energy, electron beam colliding with antiprotons, can induce a tuneshift on the antiproton bunches, which has the opposite sign to that, which they experience, from the protons. With appropriate choice of parameters two such lenses could provide effective beambeam tuneshift compensation. An R\&D program has resulted in the construction and, recently, the successful testing of a single such device. If results continue to be positive the use of such devices could lead to a longer luminosity lifetime in the Tevatron and hence to a large integrated luminosity. Another potential luminosity improvement may come from compensation of non-linear tune spread within each antiproton bunch (footprint compression) by using electron beams with Gaussian profiles [2].

Fig.1 depicts a general layout of the TEL. The magnetic system of the TEL (see details in [3]) consists of a $65 \mathrm{kG} \mathrm{SC}$ main solenoid, four $8 \mathrm{kG}$ and two $2 \mathrm{kG} \mathrm{SC}$ dipole correctors in the same cryostat, and $4 \mathrm{kG}$ gun and collector solenoids. The TEL cryostat is part of the Tevatron magnet string cooling system. A strong Пshaped magnetic field is needed to guide $10 \mathrm{kV}$ electron beam from an electron gun thru an interaction region, where electrons collide with high energy (anti)protons, to a water cooled collector. SC dipole correctors allow precise steering in position and angle of the electron beam onto the Tevatron beams. A number of precautions have been taken during SC magnets fabrication in order to achieve very high linearity of magnetic field lines inside the main solenoid. The reason is that as the electron beam goes along magnetic field lines it should not deviate around the straight Tevatron beam trajectory, otherwise the effectiveness of the TEL would be deteriorated.

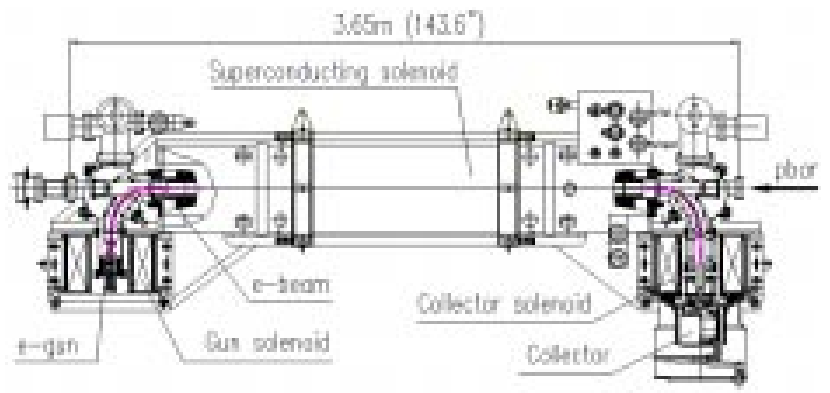

Figure 1: General layout of the Tevatron Electron Lens.

Measured rms deviations of the lines are $15 \mu \mathrm{m}$ in the vertical plane and $50 \mu \mathrm{m}$ in the horizontal plane (which is the plane of the bends) [4]. This is $10 \%$ of the Tevatron beam size in the location of the electron lens. It was found experimentally that the electron beam can be steered to pass through the main solenoid if the gun solenoid field is in the range of $B_{\text {Gun }}=1.9-4.2 \mathrm{kG}$ for $B_{m}=35 \mathrm{kG}$ (outside the range, the beam touches parts of the vacuum system) [5].

The electron gun employs a $10 \mathrm{~mm}$ diameter convex thermo-cathode and can provide up to $6 \mathrm{~A}$ of pulsed current and $3 \mathrm{~A}$ DC of up to $15 \mathrm{kV}$ electrons. Perveance of the gun is $5.6 \mu \mathrm{A} / \mathrm{V}^{3 / 2}$. Electron current profile is close to rectangular, but can be changed to a more smooth one if a negative potential (w.r.t. the cathode) is applied to a special near-cathode electrode. Water cooled collector is characterized by high-perveance of about $10 \mu \mathrm{A} / \mathrm{V}^{3 / 2}$, high absorbing efficiency exceeding 99.5\%, and dissipation up to $50 \mathrm{~kW}$ of electron beam power. See details on the gun and collector in [5].

In order to vary electron current at the scale of the bunch-to-bunch spacing (396ns in the Tevatron at present Run IIa) high-voltage pulses are applied to the gun anode. During the first studies a $8 \mathrm{kV}, 800 \mathrm{~ns}$ FWHM modulator based on RF tube has been used to provide electron pulses synchronized with a single Tevatron bunch at the repetition frequency of $47.7 \mathrm{kHz}$ (see [6] for details).

The TEL is equipped with 4 BPMs: one vertical and horizontal at the beginning and at the end of the main solenoid. The BPMs are supposed to measure transverse positions of electron, proton and antiproton beams passing through and thus, allow the electron beam to be centered on the antiproton or the proton one. $100 \mu \mathrm{m}$ diameter tungsten wires, vertical and horizontal, can be introduced into the very middle of the interaction region for electron current profile measurements. They are remotely controlled and removed when high energy beams circulate in the machine. Electron currents leaving the cathode, into the collector and onto the collector entrance electrode are 
measured by 3 inductive coils. There are $10 \mathrm{HV}$ electrodes around the electron beam trajectory which can be used for ion or secondary electron cleaning (though most of the time there are grounded). Beams diagnostics employed in the TEL is described in detail in [7].

The TEL vacuum under working conditions with 3 ion pumps with a total pumping speed of $300 \mathrm{l} / \mathrm{s}$ ranges from 4 to 10 e- 8 Torr. Table 1 summarizes main parameters of the TEL.

\begin{tabular}{|l} 
Table 1: TEL operational parameters \\
\begin{tabular}{|lll|}
\hline electron beam energy, & $U_{e}, \mathrm{kV}$ & $6-12$ \\
maximum peak electron current & $J_{e}, \mathrm{~A}$ & $2-3.5$ \\
magnetic field in main solenoid & $B_{m}, \mathrm{kG}$ & 35 \\
\multicolumn{1}{|c|}{ in gun solenoid } & $B_{g}, \mathrm{kG}$ & 3.7 \\
e-beam radius in main solenoid & $a_{e}, \mathrm{~mm}$ & 1.75 \\
cathode radius & $a_{c}, \mathrm{~mm}$ & 5 \\
e-pulse width, FWHM & $\tau_{e}, \mathrm{~mm}$ & $\sim 800$ \\
current stability, peak-to-peak & $\Delta J_{e} / J_{e}, \%$ & $<0.1$ \\
effective interaction length & $L_{e}, \mathrm{~m}$ & 2.0 \\
valve-to-valve length & $L_{t o t}, \mathrm{~m}$ & 3.65 \\
\hline
\end{tabular}
\end{tabular}

We observed very minimal effects of the magnetic fields of the lens on $980 \mathrm{GeV}$ proton beam. Tunes are shifted by less than 0.001 in both planes, the tune split $Q_{x}-Q_{y}$ varies from 0.0072 to 0.0077 , no coupling correctors are needed to operate the Tevatron, and the orbit distortion around the ring stays within about $1 \mathrm{~mm}$. Measurements with the proton beam have shown that numerous electrodes of the TEL (BPMs, HV electrodes) and discontinuities of the beam pipe all together generate a broadband impedance $|Z / n|<0.1 \mathrm{Ohm}$, that is a very small contribution to the total Tevatron impedance estimated to be some 2-8 Ohm.

In March-April 2001 there were total of seven 8-hour beam shifts dedicated to studies with the Tevatron Electron Lens. Most experimental results were obtained with a single coalesced proton bunch in the ring at the energy of $980 \mathrm{GeV}$ and everywhere below we assume that proton energy if not specifically stated. Total proton bunch length was less then $19 \mathrm{~ns}$, bunch intensity varied from 6 to $60 \times 10^{9}$. The only shift at $150 \mathrm{GeV}$ on March 23 was the very first one and to our great satisfaction a decent betatron frequency shift was observed, breaking the path for application of electron lenses in high-energy accelerators.

\section{PROTON TUNE SHIFT DUE TO TEL}

According to [1], a perfectly steered round electron beam with a constant current density distribution will shift the betatron tune by:

$$
d Q_{x, y}=\mp \frac{\beta_{x, y}}{2 \pi} \cdot \frac{1 \pm \beta_{e}}{\beta_{e}} \cdot \frac{J_{e} L_{e} r_{p}}{e \cdot c \cdot a_{e}^{2} \cdot \gamma_{p}}
$$

where the sign reflects defocusing for antiprotons and focusing for protons, $\beta_{\mathrm{e}}=v_{\mathrm{e}} / c$ is the electron beam velocity, $\beta_{x}=101 \mathrm{~m}$ and $\beta_{Y}=28 \mathrm{~m}$ are beta functions at the location of the lens (the first TEL is installed in the Tevatron sector F48), $a_{e}, J_{e}$ and $L_{e}$ stand for the electron beam size, current and effective interaction length, $r_{p}$ is the classical proton radius, $\gamma_{p}=1044$ relativistic Lorentz factor for $980 \mathrm{GeV}$ protons. Electron beam is assumed to be much wider than (anti)proton beam, so, all high-energy particles acquire the same $d Q$. Factor $l \pm \beta_{\text {e }}$ reflects the fact that contribution of the magnetic force is $\beta$ times the electric force contribution and depends on the direction of the electron velocity. So far we operated only with protons only (while the actual goal is to operate with antipron bunches) which move in the same direction as the TEL electrons, so the magnetic force reduces the total tuneshift.

Fig.2 shows an example of the Schottky spectra of horizontal proton beam oscillations without electron current and with $3 \mathrm{~A}$ electron current. One can see that the horizontal tune is shifted positively by about $d Q_{x}$ $=+0.0065$ from 20.5824 to 20.5889 . One should expect that the same electron beam would shift the horizontal tune of antiprotons $\left(1+\beta_{e} / 1-\beta_{e}\right)=1.5$ times this amount, i.e., by -0.01 given that $\beta_{e} \approx 0.2$. Besides a central peak corresponding to the betatron frequency (highlighted by marker line), the spectra consist of several synchrobetatron sidebands, separated by the synchrotron tune $Q_{s}$ $\approx 0.0007$. Total power in the peaks depends on proton intensity and noise level exciting the beam motion. Application of the electron beam may or may not cause spectra shape variation as in Fig.2. The shape also depends on the machine tuning, working point, etc. The shape variations sometimes make precise tuneshift measurements rather difficult, and we estimate typical error to be $\delta Q \approx \pm 0.0001$.

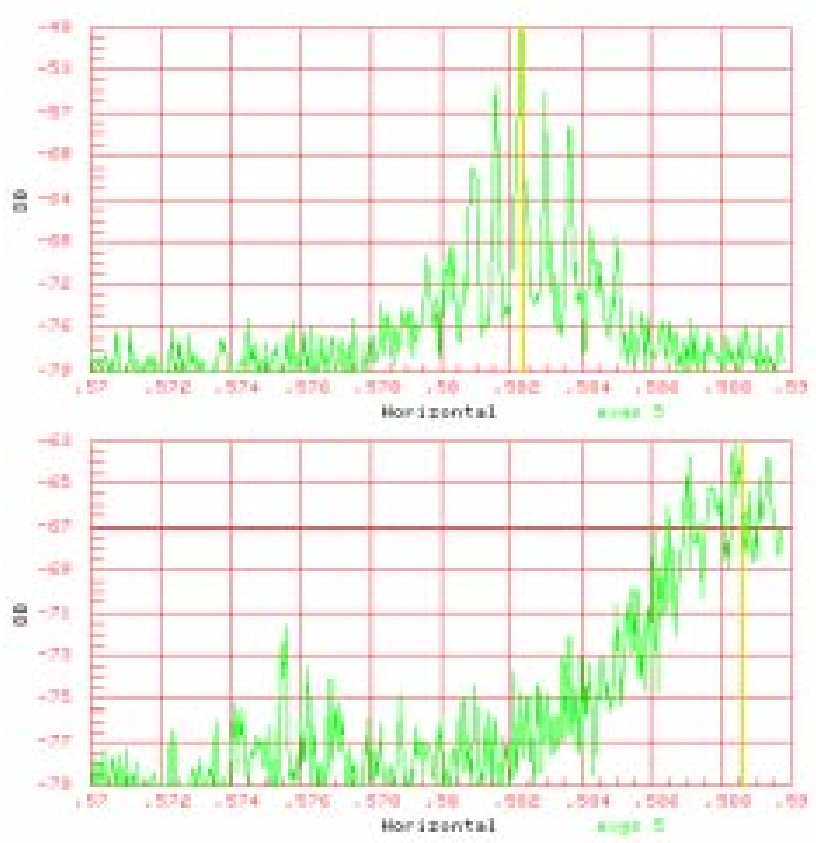

Figure 2: Schottky spectra of horizontal motion of protons without electron current (top) and with $3 \mathrm{~A}$ of electron current (bottom), cathode potential $U_{c .}=7.6 \mathrm{kV}$. 
Fig. 3 shows how the proton tune shifts depend on the time delay between the $2 \mathrm{~A}$ electron pulse and the arrival of the proton bunch. One can see that a) the tune shift follows the electron pulse shape and, therefore, it's possible to shift the tune for any bunch without touching neighbors $400 \mathrm{~ns}$ aside, and b) horizontal tune shift is some 4 times the vertical one $d Q_{x} / d Q_{y}$ $=0.0037 / 0.0008=4.6$ that is close to the beta function ratio $\beta_{\chi} / \beta_{y}=101 / 28=3.6$. The remaining discrepancy can be explained by either uncertainty in beta-functions, which is known to be $\pm 10 \%$, small ellipticity of the electron beam, mis-steering of the electron beam, which might play role if compared with $a_{e}$.

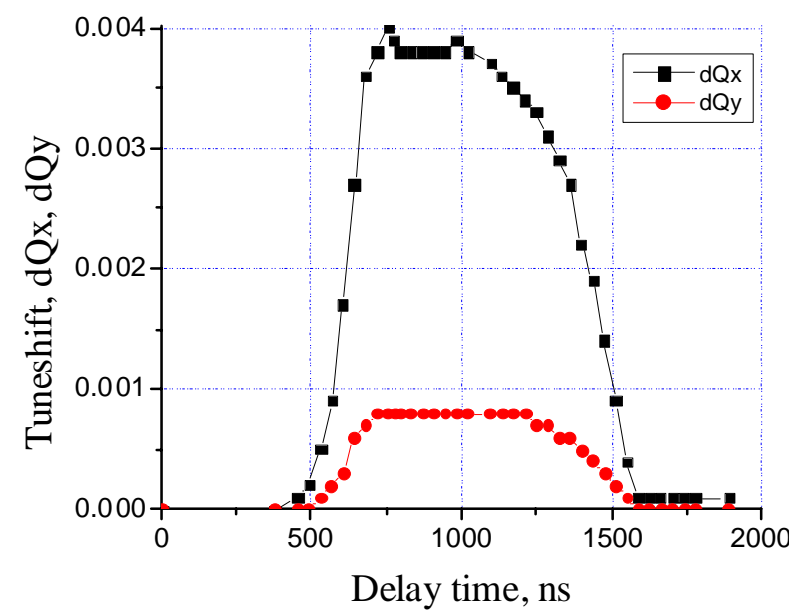

Figure 3: Shift of the horizontal (black) and vertical proton tunes vs delay between the proton bunch and 800 ns long, 1.96 A peak electron pulse, $U_{c}=6.0 \mathrm{kV}$.

Having the electron beam properly synchronized for maximum effect, we have studied dependence of $d Q_{x}$ on the peak electron current. The results are presented in Fig.4 and compared with Eq.(1). The theoretical dependence is non-linear because the electron energy inside the vacuum pipe and, thus, $\beta_{\mathrm{e}}$, goes down with the current due to electron space charge, $U_{e .}=U_{c .}-g Q_{s c}$, where $g$ is the geometry dependent factor. As seen in Fig.4, the maximum discrepancy is about $20 \%$ at $J_{e .}=2 \mathrm{~A}$. There are systematic errors in a number of parameters used for calculations, e.g., $a_{\mathrm{e}}{ }^{2}$ is known within $\pm 10 \%$, effective length $L_{e}$ depends on precision of the steering and may vary within $\pm 10 \%$, and the electron current calibration each contribute some $\pm 5 \%$ error. In addition there might be some $\pm 5 \%$ uncertainty in the electron velocity $\beta_{\mathrm{e}}$ due to formation of an ion cloud which shields some fraction of the electron space-charge $Q_{s c}$. An indication of that is that maximum electron current allowed to propagate through the beam pipe at a given cathode potential of 7.5 $\mathrm{kV}$ goes down by $25 \%$ if the pulse repetition rate is reduced from $47.7 \mathrm{kHz}$ (standard regime of operation with a single proton bunch) to about $50 \mathrm{~Hz}$. On the other hand, ions do not change charge density and thus do not contribute to $d Q$ directly most probably because of larger transverse size of the cloud.

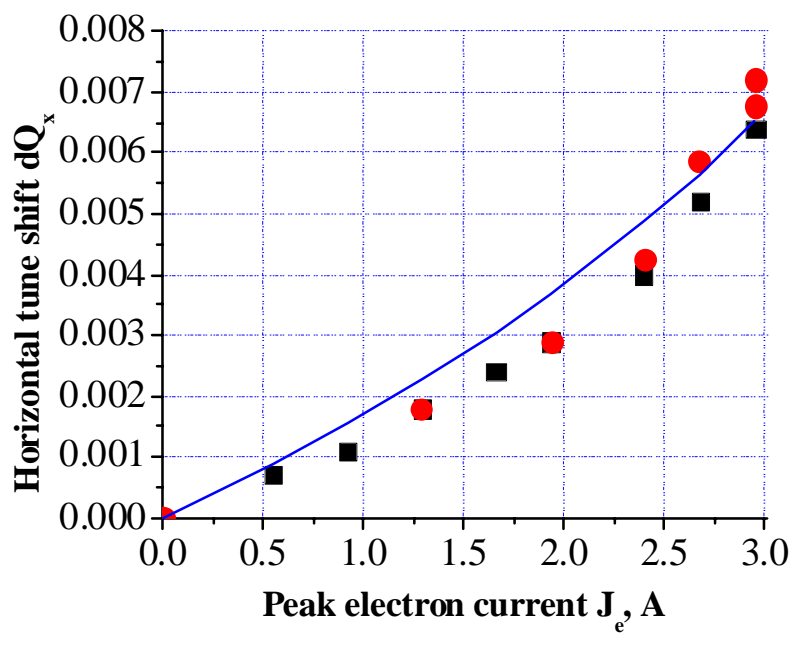

Figure 4: Shift of the horizontal proton tune vs the electron current, $U_{c}=7.5 \mathrm{kV}$. Circles and squares experimental data, solid line - Eq.(1).

It might be of interest to mention, that horizontal tune shift for protons coming just after the electron pulse (delay times from 0 to $400 \mathrm{~ns}$ in Fig.3) is slightly lower than $d Q_{x}$ for protons arriving right before the electron beam enters the interaction region (delay times above $1600 \mathrm{~ns}$ ). The little difference of about -0.0001 electrons (if one believes that it is not just a measurement error) can be associated with defocusing effect due to ions freshly attracted inside the electron beam.

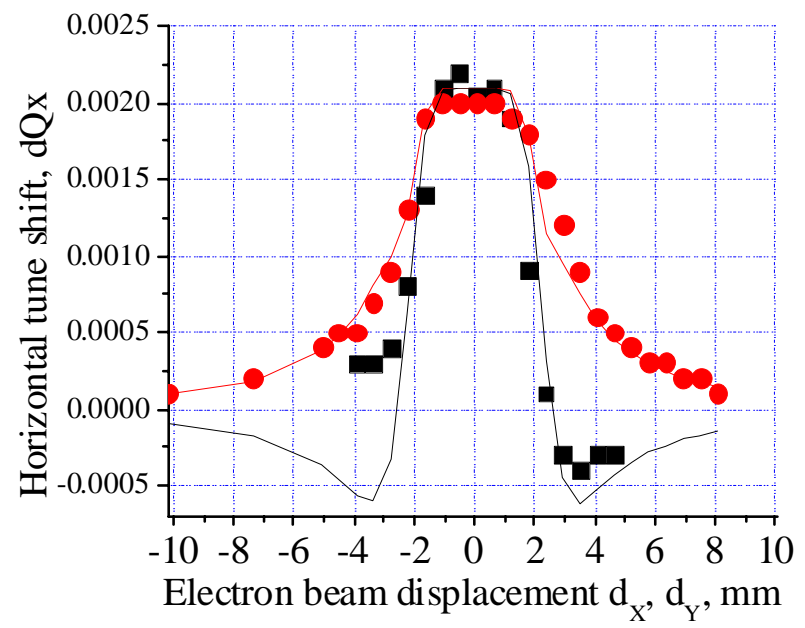

Figure 5: Horizontal tune shift vs horizontal (squares) and vertical (circles) displacement of the electron beam, $J_{e}$ $=1 \mathrm{~A}, U_{c}=6.0 \mathrm{kV}$.

As long as the proton beam travels inside a wider electron beam, the proton tune shift does not depend much on the electron beam position, e.g., for the case $1 \mathrm{~A}$ electron beam $d Q_{x}\left(d_{x}, d_{y}\right) \approx d Q_{\max }=0.0021$ if $\left|d_{x, y}\right|<2 \mathrm{~mm} \mathrm{-}$ 
see Fig.5. But when distance between centers of the two beams exceeds the electron beam radius then one should expect $d Q_{x}\left(d_{x} d_{y}=0\right) \approx-d Q_{\max } /\left(d_{x} / a_{e},\right)^{2}, \quad\left|d_{x}\right|>>a_{e}, \quad$ and $d Q_{x}\left(d_{x}=0, d_{y}\right) \approx+d Q_{\max } /\left(d_{y} / \mathrm{a}_{\mathrm{e}},\right)^{2}\left|d_{y}\right|>>a_{e}$ (note the sign). Theoretical predictions $d Q_{x}\left(d_{x} d_{y}\right)-$ see smooth curves in Fig.5 - are in a good agreement with experimental data. The only visible discrepancy is an asymmetry in $d Q_{x}\left(d_{x}, d_{y}=0\right)$. At negative horizontal displacements, $d_{x, y}<-$ $2.5 \mathrm{~mm}$, the tuneshift does not change sign as it does at $d_{x, y}>+2.5 \mathrm{~mm}$. The effect is, most probably, due to the asymmetric П-shape of the electron beam (see Fig.1), which results in additional positive contribution to $d Q_{x}$ from the bending portions of the beam if the protons propagate through them.

To summarize, we can say that experimentally observed tuneshifts agree reasonably well with theory.

\section{BEAM LIFETIME WITH TEL}

There is no formula to estimate the (anti) proton beam lifetime $\tau=(d N / d t / N)^{-1}$ under impact of the TEL. Nevertheless, analytical studies [2] and numerical tracking [8] predict the following phenomena concerning the lifetime: 1) $\tau$ depends on non-linear resonances in the vicinity of the machine working point; 2) it should decrease if the electron beam is mis-steered and protons experience non-linear forces of the electron beam; but if the beam-beam separation is very large, then the electron beam should not affect the lifetime; 3 ) one should expect better lifetime for the same $d Q$ if the electron beam is wider and its current density profile is a smooth, bell-like function.

We found that without collisions the Tevatron proton beam lifetime is very good over a broad range of the beam parameters and the machine working points (WP) $Q_{x}, Q_{y}$. Because of the limited time of the studies, we measured lifetimes based on 15 minutes records of the beam intensity records. This resulted in some $50 \%$ error in $1 / \tau$ when typical lifetime was some $\tau_{0}=90$ hours.

Collisions with the multi-Ampere electron beam did always cause certain deterioration of the $\tau$, but the best lifetime was observed at good WPs. Fig.6 shows the set of resonances up to $12^{\text {th }}$ order over the range $Q_{x, y}=20.55$ 20.60 which is typical for the Tevatron collider operation. Arrows represent the tuneshift due to the TEL. The longest one reflects the result of the very first beam study shift, the very first attempt to operate the TEL with 150 $\mathrm{GeV}$ protons. All others were obtained with $980 \mathrm{GeV}$ protons on different shifts. Numbers near each arrow show the best lifetime achieved at that WP with the maximum electron current. Electron and proton currents and beam sizes were about the same for all these observations while we can not guarantee that the electron beam was always steered with the same precision (see next Section).

One can see, that the smallest lifetimes of 1.5-6 hrs were observed when the Tevatron operated at the $7^{\text {th }}$ order resonances at $Q_{x}, Q_{y}=0.573,0.567$, better lifetimes of 6-13 hours at the $12^{\text {th }}$ order resonances $Q_{x}, Q_{y}=0.583,0.577$, and the best lifetime of 24 hours was achieved away from resonances at $Q_{x}, Q_{y}=0.564,0.555$.

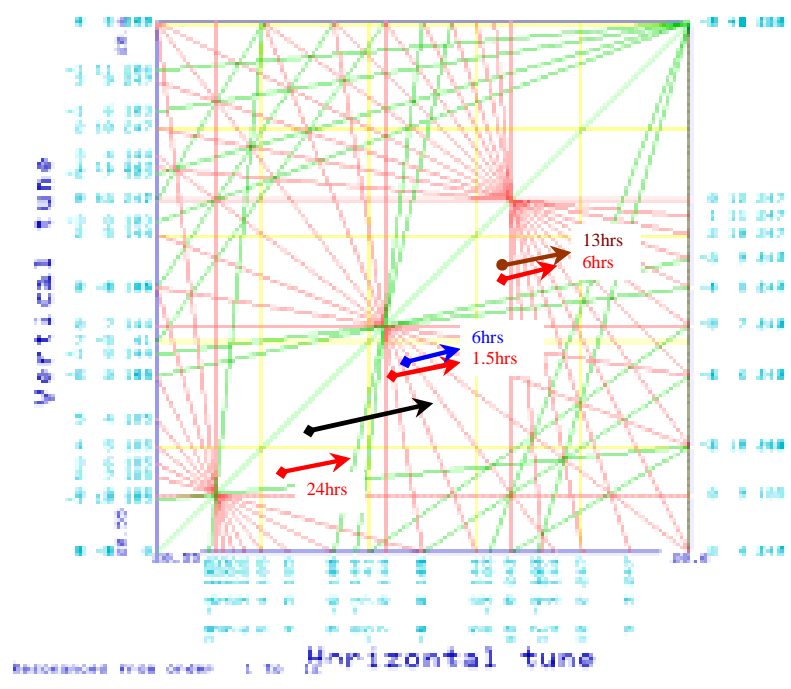

Figure 6: Proton bunch tuneshifts due to the TEL and corresponding lifetimes.

Our experience shows that mis-steering of the electron beam is by far the most important factor affecting the lifetime. It can affect $\tau$ even at comparatively small electron currents. For example, Fig.7 shows a 20 minute record of the proton bunch population (top curve) which originally was about $2.6 \mathrm{e}+10$ and the average electron beam current which was constant at $35 \mathrm{~mA}$

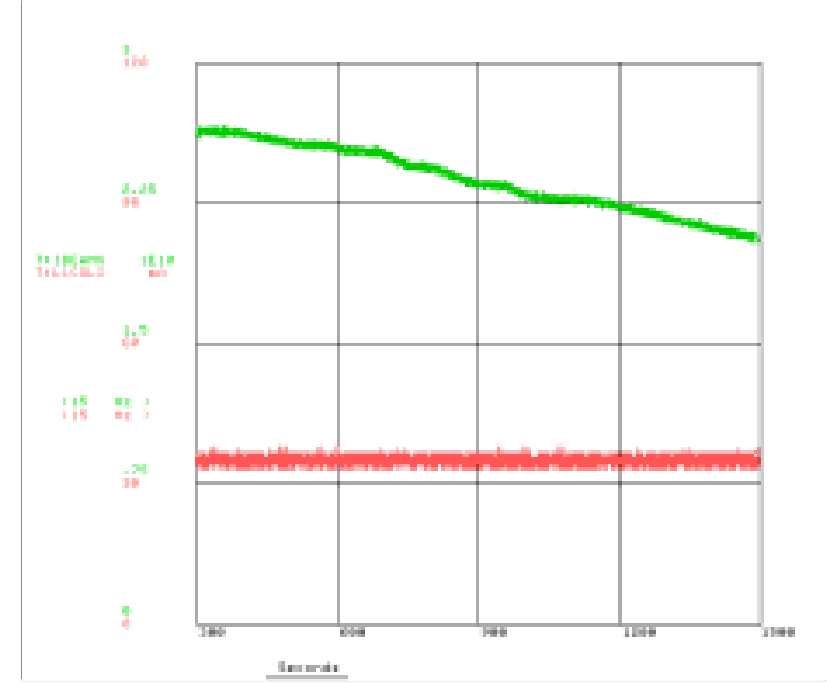

Figure 7: Proton beam intensity and electron current during the electron beam steering.

The record was taken during the measurement of the tuneshift vs the electron beam position presented in Fig.5 $\left(J_{e}=1 \mathrm{~A} \quad U_{c}=6.0 \mathrm{kV}\right)$ at the "better WP" of $Q_{x}, Q_{y}=0.583,0.577$. One can see that intensity does not go down smoothly, drops occur while we cross the electron beam edges. One may associate these with excitation of non-linear resonances. At very large electron currents we also detected significant proton emittance blow-up, which sometime made a good lifetime impossible after that. 
On the other hand, if electron and proton beams are separated by some $5 \mathrm{~mm}$ (about 3 times the electron beam radius $a_{e}$ ), than no deterioration of the proton beam intensity has been observed and the measured lifetime is about $\tau_{0}$.

We did not have enough time to study the effect of the electron beam size and/or electron current density profile yet. The only indication that relative size matters is that when the proton emittance is 1.5-2 times larger than usual, e.g. $40-60 \pi \mathrm{mmmrad}(95 \%)$ instead of $25 \pi$ mmmrad (corresponding to a rms horizontal beam size at the TEL location of $0.8-0.9 \mathrm{~mm}$ instead of typically 0.7 $\mathrm{mm}$ - compare with $a_{e}=1.75 \mathrm{~mm}$ ), the lifetime becomes very poor.

\section{FUTURE STUDIES, IMPROVEMENTS}

Topics for our further studies include: effects of the electron beam size and shape on the tuneshift and lifetime, emittance growth vs electron beam current and position stabilization, effects of ions, TEL operation with the Tevatron antiproton beam, and, finally, the TEL operation with many bunches. The ultimate goal of the studies is to achieve the same or better beam lifetime with the TEL at $d Q$ comparable with the Tevatron beam-beam tune shift and around typical working points.

Further studies will require (in order of urgency): better electron beam steering, better proton beam diagnostics, and better quality electron beam. To achive more precise steering we are currently working on the BPM hardware and electronics improvement (the existing ones gave unreliable readings of the proton bunch position.

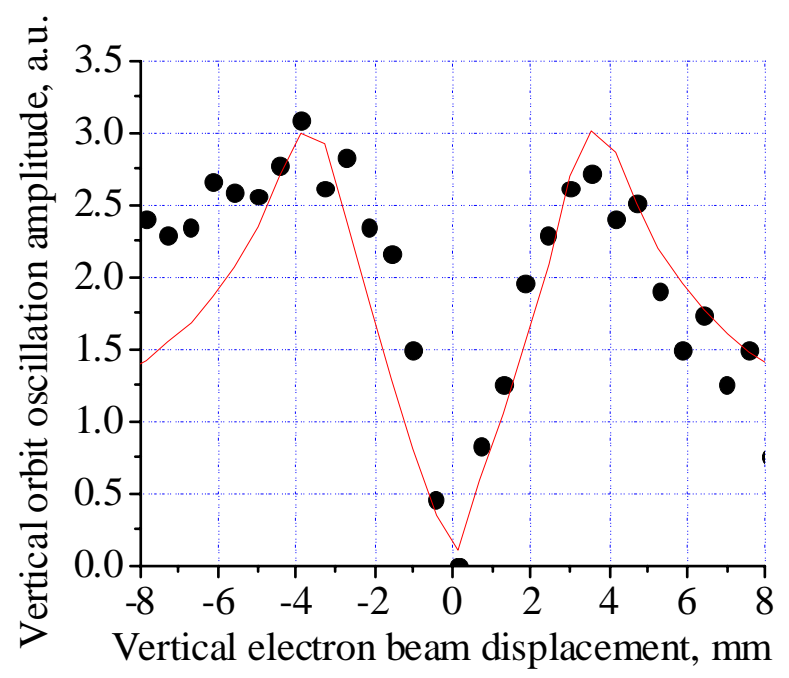

Figure 8: Rms amplitude of vertical proton orbit variation vs vertical position of AC electron beam.

Using "tickling" of the proton orbit with the electron beam can potentially improve the steering as well. The idea is similar to the "K-modulation" in the beam based alignment: variation of the electron current in the electron lens should cause variations in the proton beam orbit around the ring if the electron lens beam is not centered. Fig. 8 shows the rms amplitude of the vertical proton orbit variation at the Tevatron BPM located at A0 sector vs vertical displacement of the electron beam at F48 which had the current modulation of $J_{e}=1.02+$ $0.18 \sin \left(2 \pi t^{*} 107 \mathrm{~Hz}\right) \mathrm{A}$. The amplitude becomes equal to 0 if the proton beam goes through the center of the electron beam. The $7 \mathrm{~mm}$ distance between the two peaks reflects an effective diameter of the electron current distribution, and, thus, indicates angular misalignment of the electron beam because it exceeds the electron beam diameter of about $3.5 \mathrm{~mm}$. Therefore, steering by the orbit tickling should concentrate not only on the search of the minimum orbit response, but also on having two maxima closer to each other. In the first experiments, such a tickling measurements took about 2-3 hours, and now we are looking for a faster automated system.

We also look forward to having more reliable proton diagnostics for the emittance measurements (e.g., synchrotron light system instead of flying wires) and an automated tune measurement system for the multi-bunch measurements. R\&D on the better electron beam for the TEL include a wider beam with smooth edges from new $10 \mathrm{~A}, 30 \mathrm{kV}$ electron gun pulsed by solid-state HV FIDpulser [6], and a better stabilization of the beam current and position.

\section{CONCLUSION}

We have experimentally demonstrated feasibility and operation of an electron lens. Experimental studies of the beam-beam effects in the electron-proton collisions have been performed and shown a decent agreement with theory. We will continue our studies on the beam-beam compensation with TELs. We found no "show-stoppers" yet, and we know what we have to do on each of the problems.

Besides linear and non-linear BBC, electron lenses - a novel type of accelerator element - can be used for cleaning, dumping and shaping high-energy beams, for space-charge compensation in low-energy proton boosters [9], for slow extraction from particular bunches [10], for increasing transverse impedance and TMCI studies.

We sincerely thank all the people who contributed to the TEL design, fabrication, commissioning, and operation.

\section{REFERENCES}

[1] V.Shiltsev, et.al, Phys.Rev. ST-AB, 2,071001(1999)

[2] Y.Alexahin, et.al, these Proceedings.

[3] A.Ageev, et.al, these Proceedings.

[4] K.Bishofberger, et.al, these Proceedings.

[5] N.Solyak, et.al, these Proceedings.

[6] D.Wildman, et.al, these Proceedings.

[7] N.Solyak, et.al, these Proceedings.

[8] D.Shatilov, et.al, these Proceedings.

[9] A.Burov, et.al, these Proceedings.

[10] V.Shiltsev, J.Marriner, these Proceedings. 
